\title{
INVESTIGACIÓN
}

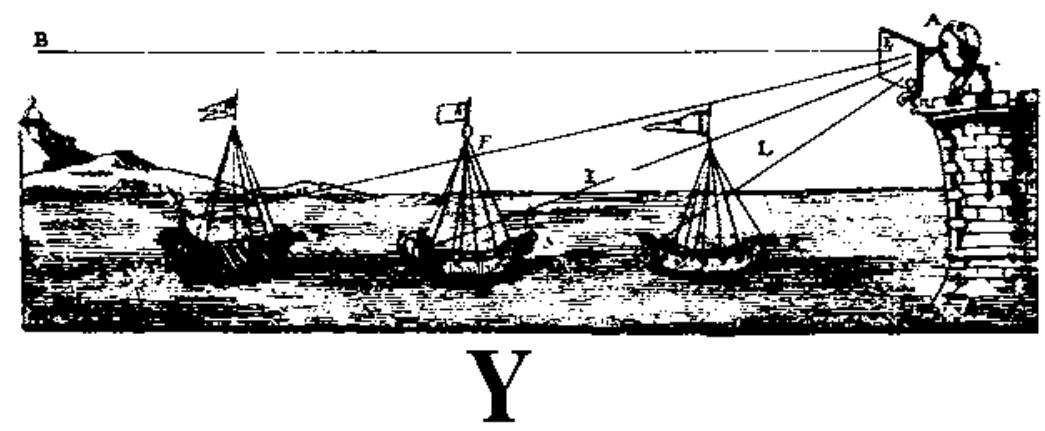

EXPERIENCIAS
DIDÁCTICAS

\section{EL PARADIGMA DE LAS CONCEPCIONES ALTERNATIVAS Y LA FORMACIÓN DE LOS PROFESORES DE CIENCIAS}

\author{
VÁZQUEZ ALONSO, A. \\ Servicio de Inspección de Educación. Pasaje Guillermo Torrella, 2, planta 6. 07002 Palma de Mallorca.
}

\section{SUMMARY}

The pre-service education of science teachers is empirically studied in the framework provided by the paradigm of alternative conceptions by means of 13 simple usual Physics questions. The rates of pre-service teachers' alternative conceptions show a strong resemblance with those found in previous research even with students. Differences between sex and age groups are remarkable, but the major finding is that only physicists show a suitable pattern lacking alternative conceptions about Physics topics, while other science careers not only have a lot of alternative conceptions but also science and non-science teachers show similar patterns. We claim a pre-service teacher education more based on conceptual change aimed to avoid teachers' alternative conceptions in basic science contents of different areas.

\section{EL PARADIGMA DE LAS CONCEPCIONES ALTERNATIVAS Y LA FORMACION DELOS PROFESORES DE CIENCIAS}

Desde que se inició el estudio sistemático de los grandes errores de los alumnos (respuestas incorrectas científicamente) cuando responden a cuestiones básicas relacionadas con los aprendizajes de ciencias realizados (Viennot 1979), la investigación sobre concepciones alternativas se ha convertido en un tema central de la investigación en didáctica de las ciencias. Paradóficamente, a pesar de esta importancía, no existe unanimidad sobre la denominación de este fenómeno. Las denominaciones más empleadas son "esquemas conceptuales" 
(Cubero 1988, Champagne, Gustone y Klopfer 1983), «esquemas conceptuales alternativos» (Driver y Easley 1978), «teorías ingenuas» (Caramazza, McCloskey y Green 1981), “concepciones alternativas» (Hewson 1981), "ciencia de los niños», «ciencia intuitiva» (Osborne Bell y Gilbert 1983), «errores conceptuales»-misconceptions(Helm 1980), «preconceptos»-supersticiones, creencias infundadas, conocimiento incidental- (Carrascosa y Gil 1987), «ideas previas» (Hierrezuelo y Montero 1989), «concepciones espontáneas» (Pozo y Carretero 1987), etc. Aquí emplearemos la denominación de concepciones alternativas, de acuerdo con el razonamiento y la justificación de Abimbola (1988).

Una de las características más importantes de las concepciones alternativas es su persistencia en la mente de quienes las sustentan. Esta persistencia supone que la mayoría de los individuos que no han estudiado ciencia las mantienen toda su vida como parte del sistema de creencias sobre la naturaleza (ciencia intuitiva), pero, desde el punto de vista educativo, lo más llamativo es que el estudio de la ciencia no logra, en muchos casos, removerlas, de manera que estudiantes universitarios, e incluso profesores en ejercicio, muestran tasas de errores elevadas (Sebastià 1984). Parece que esta resistencia al cambio conceptual de ias concepciones alternativas es más importante en aquellas cuestiones relacionadas con hechos y fenómenos que los alumnos observan con frecuencia. Para explicar la persistencia de las concepciones alternativas en la estructura mental de los sujetos se han aducido numerosos factores, como la metodología de la superficialidad (Carrascosa y Gil 1985), que impulsa respuestas rápidas, seguras y acríticas. En esta misma línea de conducta epistemológicamente deficiente, se señala también la tendencia a considerar sólo las pruebas que confirman una tesis y olvidar las que la contradicen (como un rasgo psicológico que favorece el equilibrio emocional, porque la falsación supondría conflicto), incluso considerando que las excepciones «confirman la tesis» (Hashwed 1986). Además, una educación científica que no tiene en cuenta las concepciones alternativas de los alumnos, tanto antes como después del aprendizaje, contribuye decisivamente a su mantenimiento.

Otra característica muy importante de las concepciones alternativas es su relativa coherencia interna en cada persona. No se trata, pues, de rasgos oportunistas o puntuales, ad hoc para una situación particular, sino verdaderas estructuras teóricas jerarquizadas, generalmente implícitas, que cumplen una función explicativa y que permiten al individuo controlar los acontecimientos diarios de su ambiente. Driver y Erickson (1983) las denominan teorías-en-acción, es decir, estructuras cognitivas que permiten predecir correctamente los sucesos, pero cuya verdadera naturaleza «incorrecta científicamente» queda implícita u oculta porque la predicción, sobre todo si es exitosa, no suele ir acompañada de una explicación que podría revelar su alcance incorrecto.

Las concepciones alternativas están muy extendidas, en diferentes medios y en sujetos de diferentes edades, jóvenes y adultos; estudiantes con años de estudios científicos a sus espaldas muestran resultados muy pare- cidos a otros cuyo contacto con la ciencia formal es menor. Estudiantes universitarios, licenciados, e incluso profesores (Fernández y Vázquez 1991, Nussbaum 1981, Vázquez 1990) exhiben claramente estas concepciones alternativas, sugiriendo un cierto grado de inconsciencia en su mantenimiento. El caso más típico son los estudiantes que ofrecen la respuesta convencional y adecuada de la ciencia a un problema, cuando se encuentran en situación de «dar la respuesta correcta» y exhiben concepciones alternativas cuando expresan sus ideas en otro contexto, alejado de la clase de ciencias. Las concepciones alternativas son un rasgo importante que revela la incapacidad de la enseñanza tradicional de las ciencias para conseguir el cambio conceptual y lograr aprendizajes significativos, pero no se pueden atribuir a ellas todas las dificultades de los alumnos en el aprendizaje de las ciencias. Sin embargo, sus hallazgos y conclusiones configuran la base de un nuevo modelo didáctico (constructivista) para el aprendizaje de las ciencias (Gil 1987).

Adicionalmente, las concepciones alternativas poseen un carácter histórico, ya que reproducen ideas y concepciones públicamente admitidas en etapas históricas precedentes y que la evolución del conocimiento científico ha enterrado. Según de Vega (1983), el hecho de que las concepciones alternativas sean compartidas por muchos individuos contemporáneos y a través de los siglos y en distintos ambientes culturales podría ser debido a las restricciones que nuestra naturaleza biológica impone al procesamiento de la información.

Algunas de las principales concepciones alternativas en ciencias han sido revisadas, resumidas y sistematizadas en diversos estudios (Driver y Erickson 1983, Helm y Novak 1983, McDermott 1984, Osborne y Freyberg 1985 , Driver, Guesne y Tiberghien 1989, Hierrezuelo y Montero 1988).

Las concepciones alternativas no sólo se generan mediante el razonamiento natural; la investigación sobre las mismas ha puesto de relieve que el propio sistema escolar puede ser fuente ellas (Helm 1980, Lehrman 1982) a través de los errores en libros de texto y de un aprendizaje inadecuado, y en todo caso, constituye una llamada de atención sobre la ineficacia de la enseñanza tradicional de las ciencias.

La exhaustiva investigación sobre las concepciones alternativas de los alumnos, cn todas las áreas y niveles de enseñanza y en diversos países y culturas, ha generado una importante cantidad de información dirigida principalmente al diagnóstico e identificación de estas concepciones. Aunque la presencia de las concepciones alternativas, incluso en alumnos universitarios de la especialidad de ciencias, es un resultado evidenciado en la investigacion, tal vez no se ha insistido suficientemente en la trascendencia de este resultado para la enseñanza de las ciencias, ya que algunos de estos alumnos universitarios se convierten en profesores. El objetivo de este trabajo es analizar esta relación.

La selección de los profesores de enseñanza secundaria de las asignaturas de ciencias se realiza entre los titula- 
dos superiores; sin embargo, aunque existe una cierto grado de coincidencia entre especialidad de título y asignatura impartida, por diversos motivos también se producen frecuentes desajustes:

- En primer lugar, porque las asignaturas en secundaria y bachillerato no coinciden exactamente con las titulaciones declaradas prioritarias (concordantes) para su desempeno; éste sería el caso de física o química cuyo profesorado concordante son licenciados en Físicas y licenciados en Químicas, y también de ciencias naturales, biología o geología cuyo profesorado concordante son licenciados en Biológicas y en Geológicas; $y$ en la educación secundaria obligatoria de la reforma educativa las ciencias de la naturaleza, cuyo profesorado potencial son todas las titulaciones citadas.

- En segundo lugar, en ausencia de profesores concor. dantes, por desequilibrio entre oferta y demanda del mercado de trabajo, todas las titulaciones de ciencias son consideradas idóneas para impartir docencia de cualquier asignatura del área. Esto hace que licenciados en Bíología impartan física o matemáticas, y licenciados en Química impartan geología o biología.

En términos coloquiałes, esta situación se conoce como la problemática de la asignaturas afines, es decir, la situación de los profesores que imparten asignaturas que no concuerdan con la especialidad de titulación superior. Obviamente, esta situación conduce a una depreciación de la tarea específicamente científica que los profesores de ciencias pueden realizar en el aula, y perjudica directamente fa calidad de la enseñanza de las ciencias.

Aunque nunca explícita, una cierta justificación de esta situación participaría de la creencia de que un profesor licenciado tiene una formación suficiente para impartir Ia docencia en cursos de secundaria en cualquiera de las displinas afines a su formación superior. El objetivo de este estudio es demostrar que este argumento no es correcto: las titulaciones superiores no concordantes no suministran a los profesores una formación adecuada para las asignaturas afines de ciencias, ni siquiera para impartir los conceptos más elementales de las ciencias. Para ello, se considera que el paradigma de las concepciones alternativas ofrece un marco teórico apropiado para plantear este problema, examinar los resultados y contrastar la hipótesis adelantada.

\section{MÉTODO}

\section{Muestra}

Los participantes en este estudio han sido dos grupos de profesores en formación inicial, inscritos en un curso de diseño curricular para la formación de profesorado, con un total de 118 profesores-alumnos. Una parte de ellos son alumnos de último curso para su graduación universitaria y otros son ya titulados superiores, en diversas especialidades: Físicas (10), Químicas (20), Biológicas y afines -Geología, Agrónomos- (41), Ciencias de la
Salud-Međicina y Veterinaria-(7), Técnicos-Informática- (6), Económicas (11) y Psicología (23)-. La media de edad del grupo es de 25,3 años (desviación 4,2 , mediana, 24) con una moda de 22 , y una edad máxima de 40 y mínima de 21 . Entre los 118 profesores alumnos hay 50 hombres y 68 mujeres.

\section{Procedimiento}

A propósito del concepto de evaluación inicial, en el marco de un diseño curricular, se enfatizó la función de la misma para contribuir a la identificación de las ideas previas de los alumnos, antes de comenzar el aprendizaje. En este aspecto, se explicó la importancia extraordinaria de las concepciones alternativas en la enseñanza de las ciencias, y para demostrar en vivo la universalidad de su existencia, y como una forma de evaluación inicial, se propuso a los alumnos participar en una experiencia en la que ellos mismos iban a explicitar sus propias concepciones alternativas sobre temas básicos de ciencias. Los resultados sirven para demostrar la tesis anterior, y fueron la base para la discusión y el trabajo en clase del grupo.

Dado que los temas de física son los que más atención han recibido en la investigación de concepciones alternativas, y por la escasez de titulados en esta especialidad, se tomaron estos temas como tópicos parael diagnóstico de la evaluación inicial. Para ello los profesores contestaron en unos minutos 13 preguntas sobre estos tópicos.

\section{Instrumento}

Los profesores-alumnos respondieron 13 cuestiones de física, breves, extraídas de algunos estudios sobre concepciones alternativas, muy simples, ya que sólo pretenden diagnosticar la existencia o no de una concepción alternativa.

La cuestión 1 es una máquina de Atwood con dos pesos exactamente iguales, en una situación inicial en la que una de las pesas está más alta que la otra. Se pregunta cuál será la situación después de dejarlo en libertad (Viennot 1979). La cuestión 2 consiste en un cuerpo ascendiendo por un plano inclinado a partir de un impulso inicial. Se pregunta cuál es la dirección de la fuerza que actúa sobre el cuerpo cuando éste asciende (Carrascosa y Gil 1982). La cuestión 3 es un circuito de corriente continua con dos ramas en paralelo, con una bombilla y una resistencia iguales en cada rama (Cohen, Eylon y Ganiel 1983); se pregunta qué ocurrirá con la d.d.p.entre las bornas de una bombilla cuando ésta se quita de su casquillo (aumenta, disminuye o queda igual). La cuesrión 4 (Sebastià 1984) pregunta el diagrama de fuerzas que actúan sobre una pelota ascendiendo, en caída libre, desde un impulso inicial, of reciendo cinco respuestas alternativas. La cuestión 5 plantea la comprensión del principio de inercia y la composición de movimientos, con un tornillo que se desprende del techo del vagón de un tren en movimiento uniforme; se pregunta dónde chocará con el suelo (Whitaker 1983). La cuestión 6 plantea la comprensión de la composición de movimientos: en un disparo horizontal simultáneo dejando caer 
verticalmente una bola de hierro desde la misma altura, ¿cuál llega antes al suelo? (Whitaker 1983). La cuestión 7 plantea el problema de diferenciar entre fuerza y velocidad: ¿actúa la misma o diferente fuerza sobre 6 cuerpos iguales situados a la misma altura aunque describiendo trayectorias diferentes? (Viennot 1979). La cuestión 8 plantea tres interacciones carga-carga, tierraave e imán-hierro, preguntando ¿quién atrae a quién? (Hicrrezuelo y Montero 1988). Desde el punto de vista cuantitativo, esta cuestión equivale a tres cuestiones, para cada uno de los tres casos propuestos $(8,9$ y 10$)$. La cuestión 11 plantea el giro de la Luna en torno a la Tierra como un movimiento circular y pregunta cuál cs el diagrama de fuerzas que actúan sobre la Luna (Acevedo, Bolx́var, López-Molina y Trujillo 1989). La cuestión 12 plantea la conservación de la energía mecánica en presencia de rozamiento en el caso de un coche que afronta, con el motor parado y una cierta velocidad, una cuesta, llegando arriba, donde queda detenido; se pide discernir la frase más correcta, entre 4 propuestas (Hierrezuelo y Molina 1990). Estrictamente, la respuesta más correcta sería la que aplica el principio de conservación teniendo en cuenta cl rozamiento (criterio que se ha aplicado al cálculo de las puntuaciones individuales), y en menor medida, la que deduce de la aplicación general del principio. La cuestión 13 plantea la comprensión del concepto de densidad de un cuerpo, preguntando si es posible inferir cuál de dos cuerpos tiene un peso mayor observando los distintos niveles del agua al sumergirlos en dos vasijas idénticas (Bullejos y Sampedro 1990).
Ninguna de las cuestiones necesita cálculos numéricos, sino sólo razonar sobre la situación planteada con las reglas más básicas de la física; en algunas cuestiones se ofrecen unas pocas respuestas alternativas bien definidas cerradas, para facilitar la interpretación de las respuestas de los alumnos. Para cada alumno, se ha obtenido una puntuación directa de aciertos, que representa el número de cuestiones acertadas (puntuación máxima de 13 puntos), y una puntuación de omisiones, que representa el número de cuestiones dejadas sin respuesta.

\section{RESULTADOS}

En primer lugar se analizan los resultados obtenidos en cada cuestión, considerando las frecuencias de respuesta en cada alternativa (Tabla I), y se comparan con los resultados obtenidos en otros trabajos de aplicación de las mismas cuestiones.

La cuestión de la máquina de Atwood (1) plantea la dificultad de comprender que las situaciones asimétricas en los sistemas pueden ser situaciones da equilibrio. La mitad de los profesores-alumnos han elegido la respuesta correcta (se mantiene el sistema en la situación inicial, aunque sea asimétrica), en tanto que casi la otra mitad han optado por la solución errónea, pero simétrica (las dos pesas a la misma altura).

Tabla I

lirecuencias y porcentajes de las distintas alternativas en cada cuestión obtenidas de las tespuestas al cuestionario de concepciones atternativas de física por profesores en formación pre-servicio (subrayada la alternativa correspondiente a la respuesta correcta).

A L TER N A T IVAS

\section{CUESTIONES OMISIÓN}

(1)

\begin{tabular}{|c|c|c|c|c|c|c|c|c|c|c|c|}
\hline 1. Măquina Atwood .......... & 1 & .8 & 1 & .8 & $55 \quad 46.6$ & $\underline{60}$ & $\underline{50.8}$ & 1 & .8 & & \\
\hline 2. Plano inclinado ............. & 1 & .8 & 26 & 22.0 & $41 \quad 34.7$ & $\underline{50}$ & $\underline{42.4}$ & & & & \\
\hline 3. Circuito ........................ & 12 & 10.2 & 22 & 18.6 & 3630.5 & 35 & 29.7 & $\underline{13}$ & $\underline{11.0}$ & & \\
\hline 4. Subida libre ................... & 1 & .8 & 4 & 3.4 & $11 \quad 9.3$ & 26 & $\underline{22.0}$ & 71 & 60.2 & 5 & 4.2 \\
\hline 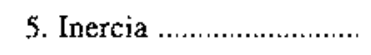 & 1 & .8 & 4 & 3.4 & $\underline{60} 50.8$ & 53 & 44.9 & & & & \\
\hline 6. Comp. movimientos .... & 3 & 2.5 & 57 & 48.3 & $\underline{46} \quad \underline{39.0}$ & 10 & 8.5 & 2 & 1.7 & & \\
\hline 7. Fuerza peso .................... & 2 & 1.7 & $\underline{49}$ & 41.5 & 6756.8 & & & & & & \\
\hline 8. Atr. electrost ................... & 6 & 5.1 & 62 & $\$ 2.5$ & $8 \quad 6.8$ & $\underline{42}$ & $\underline{35.6}$ & & & & \\
\hline 9. Atr. gravitatoria ........... & 4 & 3.4 & 77 & 65.3 & $4 \quad 3.4$ & 33 & $\underline{28.0}$ & & & & \\
\hline 10. Atr. magnética ................. & 6 & 5.1 & 80 & 67.8 & .8 & $\underline{31}$ & $\underline{26.3}$ & & & & \\
\hline 11. Fuerza circular ................ & 4 & 3.4 & 36 & 30.5 & $\underline{13} \underline{11.0}$ & 23 & 19.5 & 42 & 35.6 & & \\
\hline 12. Cons. energía ................. & 1 & .8 & $\underline{25}$ & $\underline{21.2}$ & $6 \quad 5.1$ & $\underline{67}$ & $\underline{56.8}$ & 19 & 16.1 & & \\
\hline 13. Peso y densidad ............ & 1 & .8 & 1 & .8 & $20 \quad 16.9$ & 2 & 1.7 & 94 & 29.7 & & \\
\hline
\end{tabular}


La asociación errónea entre fuerza y velocidad es una de las concepciones alternativas más comunes, en la forma de proporcionalidad entre ambas que implica a mayor fuerza mayor velocidad, pero también que la fuerza y la velocidad deben tener la misma dirección y el mismo sentido. Esta concepción alternativa se aborda en tres cuestiones diferentes $(2,4$ y 7$)$ que plantean situaciones donde fuerza y velocidad tienen dirección o sentido distintos. Sería de esperar que los alumnos que tengan clara la solución correcta deberían dar una proporción similar en las tres cuestiones; los resultados muestran que no se cumple esta hipótesis y justamente las dos cuestiones que plantean el problema en la misma situación (una caída libre) que deberían ser las más parecidas, una de ellas es la que muestra la porporción más diferente de aciertos respecto a las otras dos, lo cual evidencia el carácter extraordinariamente concreto e idiosincrático de las concepciones alternativas. Carrascosa y Gil (1982) obtuvieron un $70 \%$ de errores entre estudiantes en la cuestión 2, no muy diferente al obtenido aquí $(58 \%)$ ). En la subida libre (cuestión 4), Sebastià (1984) encontró apenas un $10 \%$ de aciertos y del orden del $80 \%$ de respuestas erróneas hacia la opción 4 (fuerza hacia arriba grande, y otra pequeña hacia abajo), resultados un poco

Tabia II

Resultados globales para la muestra de profesores en formación pre-servicio evaluados $(N=118)$ mediante el cuestionario de concepciones alternativas de Física.

VAR I A B LES

\begin{tabular}{|c|c|c|c|c|c|}
\hline \multirow{2}{*}{ Casos } & \multirow{2}{*}{$\%$} & \multicolumn{2}{|c|}{ ACIERTOS } & \multicolumn{2}{|c|}{ OMISIONES } \\
\hline & & Media & DvSt & Media & DvSt \\
\hline
\end{tabular}

\section{SEXO}

$\begin{array}{lll}\text { Hombres } & 50 & 42.4 \% \\ \text { Mujeres } & 68 & 57.6 \%\end{array}$

$42.4 \%$

$57.6 \%$

ESTUDIOS

$$
10
$$

$8.5 \%$

6

4

Químicas

Hombres

$$
\text { Mujeres }
$$

20

Hombres

Mujeres

Biología y afines

41

Hombres

Mujeres

Ciencias de la Salud

Técnicos

Económicas

Psicología

$$
23
$$

Hombres

Mujeres

$$
16.9 \%
$$

8

$34.7 \%$

12

29

$5.9 \%$

$5.1 \%$

$9.3 \%$

$19.5 \%$

20

27

\section{GRUPOS DE EDAD}

Menores de 25 años $\quad 72$

Mayores de 24 años $\quad 46$

$61.0 \%$

$39.0 \%$

Total

118

5.84

2.90

.52

1.05

3.67

2.48

.25

.69

10.20

1.47

0.00

0.00

10.50

1.51

9.75

1.50

6.05

2.48

.35

.74

6.92

2.23

4.75

2.37

3.73

2.24

.19

.71

4.67

2.35

3.34

2.13

4.14

2.41

0.00

0.00

4.00

2.60

1.00

1.26

3.63

1.50

I. 40

3.17

1.99

.81

.99

4.50

.56

2.81

2.19

1.55

4.93

3.07

.30

.83

4.06

2.46

.45

.93

4.59

2.87

.36

.87

Ninguna de las interacciones binarias entre las tres variables resulta significativa. 
peores que ios nuestros (22\% y $60 \%$, respectivamente), pero no sensiblemente diferentes. Por último, la cuestión planteada sobre el mismo asunto por Viennot (I979) con distintas trayectorias (rectas y parabólicas, subidas y caídas libres) fue respondida en un $50 \%$ diciendo que la fuerza que actuaba en los distintos casos era diferente; en nuestro caso, esta proporción es todavia mayor (casi un $57 \%$ ). Por tanto, se puede concluir que los años de instrucción de los profesores-alumnos de la muestra de este estudio, muchos de ellos graduados ya, y el resto de alumnos a punto de conseguir la graduación, en carreras científicas, no han modificado la asociación entre fuerza $y$ velocidad.

Otra faceta de la asociación entre fuerza y velocidad ocurre en los movimientos con trayectoria curva (cuestión 11), aunque aquí aparece un rasgo nuevo; la frecuente adición de fuerzas compensadoras ( «centrífugas») para explicar la dirección del movimiento. La solución correcta (una fuerza dirigida hacia el centro, alternativa 2) alcanza sólo una tasa del $11 \%$-inferior a la informada por Acevedo y otros (1989) con alumnos de $2^{\circ}$ curso de BUP-, llegando hasta un $76 \%$ la proporción de profeso. res-alumnos que eligen alguna de las alternativas (1 y 4 ) con fuerzas «centrífugas» incorporadas.

La composición de dos movimientos simples se aborda en las cuestiones 5 y 6 . El error más habitual consiste en no tener en cuenta la existencia de uno de los movimientos, sobre todo cuando el otro aparece como dominante o más rápido: en la cuestión 5 los alumnos no consideran el principio de la inercia aplicado al movimiento horizontal del tornillo-responden que cae detrás de su vertical el $45 \%$-; en la cuestión 6 , a pesar de que la pregunta enfrenta al alumno con la consideración del movimiento vertical de caída, un $48 \%$ considera que la bola llega al suelo antes que la bala. Sin embargo, la tasa de aciertos obtenida ( 59 y $31 \%$, respectivamente) es mayor que la obtenida en la aplicación del autor (Whitaker 1983).

Otro de los errores más frecuentes en relación con las fuerzas es que se las considera una propiedad de los cuerpos; esta concepción es patente cuando se conside- ran fuerzas de interacción sometidas a la tercera ley (las fuerzas son iguales en módulo y dirección, sentidos opuestos y están aplicadas sobre cuerpos distintos). Los ítems 8,9 y 10 plantean la misma cuestión para una interacción electrostática (carga $2+$ y $1-$ ), gravitatoria (tierra-ave) y magnética (imán-hierro). La primera alternativa es que el cuerpo citado en primer lugar ejerce fuerza sobre el segundo; la segunda alternativa, lo inverso de la anterior; y la tercera, que la fuerza es mutua. La mayoría de los alumnos $(52,5 \%, 65,3 \%, 67,8 \%)$ consideran la interacción asimétrica (sólo uno de los cuerpos ejerce la fuerza), sesgada hacia el cuerpo dominante por alguno de sus rasgos secundarios (tamaño de la carga 2+ o de la tierra, o mayor importancia dada al imán frente al hierro). Por otro lado, sería plausible esperar que la tasa de alumnos con la respuesta correcta fuera idéntica en los tres casos, ya que los alumnos que tuvieran claro el concepto simétrico o mutuo de las interacciones deberían contestar igual en los tres casos; sin embargo se puede observar que las tasas de respuesta correcta (alternativa 3) no son idénticas. Los alumnos con las tres respuestas correctas son $29(24,6 \%)$, en tanto que los casos con una cierta incoherencia (la fuerza es mutua ocurre sólo en uno o dos casos) son $15(12.7 \%)$.

El principio de conservación de la energía planteado en la cuestión 12 muestra que una gran mayoría (78\%) hace uso de este principio, aunque sólo un $21 \%$ tiene en cuenta la situación real con rozamiento. Estos porcentajes son muy similares a los obtenidos por los alumnos del grupo experimental de los autorcs (Hierrezuelo y Molina 1990). Análogamente, la identificación de peso y volumen al no considerar la existencia de la densidad como propiedad independiente (cuestión 13) obtiene resultados similares a los informados por Bullejos y Sampedro (1990) para su grupo experimental (tasas de aciertos del $80 \%$ ).

Por último, el circuito eléctrico en derivación planteado en la cuestión 3 sólo obtiene un $11 \%$ de respuestas correctas, siendo las alternativas 2 y 3 (incorrectas) las mayoritarias (entre ambas el 60\%); resultados extraordinariamente similares a los obtenidos por los autores (Cohen et al, 1983) con una muestra heterogénea de alumnos buenos preuniversitarios, universitarios y profesores.

Los resultados obtenidos en este estudio con profesores en formación inicial, en la mayoría de los casos, reproducen muy aproximadamente los resultados obtenidos por otros estudios citados, con muestras y condiciones diferentes; las dos últimas cuestiones (12 y 13) son las que obtienen unos resultados menos llamativos desde las perspectiva de concepciones alternativas. Se podría concluir, por tanto, que el grupo de profesores-alumnos (donde predominan profesores con formación universitaria de ciencias) muestra las mismas concepciones alternativas que otros grupos de personas.

Sin embargo, con ser valioso el análisis realizado en los párrafos anteriores, el objetivo fundamental de este estudio es comparar los resultados de los distintos grupos de profesores-alumnos, según la especialidad de sus estudios.

La tabla II contiene la media de las puntuaciones de aciertos y omisiones obtenidas por los distintos grupos de profesores-alumnos, según el sexo, la especialidad de los estudios o la edad de la muestra (mayores de 24 años y menores o iguales a 24 años), cuya comparación, para determinar el grado de significación de las diferencias, se ha realizado mediante sendos análisis de la varianza (ANOVA).

Para las puntuaciones de omisiones, es decir, las cuestiones sin respuesta, no se obtienen diferencias significativas ni entre hombres y mujeres, ni entre los dos grupos de edad; aunque sí existen diferencias entre las distintas especialidades $(p[111,6]=.0000)$, lo cual es un indicio de la importancia de la variable especialidad.

Puesto que las omisiones se pueden deber al azar, la puntuación de aciertos que analizaremos con más detalle tiene mayor importancia. El grupo de profesores-alumnos hombres (5.84) obtiene puntuaciones mejores que 
las mujeres (3.67), resultando estadísticamente significativas (grado de significación p[116,1]=.0000). Análogamente, los profesores-alumnos del grupo de mayor edad (mayores de 24 años) obtienen puntuaciones inferiores al grtupo más joven (menor de 25 años), aunque la significación de las diferencias $(p[116,1]=.1107)$ no liega al nivel mínimo usual de significación $(\mathrm{p}=.05)$, aunque está muy próxima.

Cuando se considera como variable de agrupamiento la especialidad de los estudios realizados (7 grupos diferentes), el ANOVA global resulta estadísticamente significativo (grado de significación p $[111,6]=.0000$ ), lo cual indica la existencia de diferencias significativas entre los pares de grupos. Para determinar los pares de grupos que resultan responsables de las diferencias encontradas, se ha complementado el ANOVA con el test de Scheffé (para un nivel de significación $=.05$ ) que compara las diferencias entre todas las parejas de grupos posibles. El resultado de este test muestra que el grupo de Físicas tiene una puntuación significativamente más alta que todos los demás grupos y el grupo de Químicas tiene una puntuación significativamente más alta que los de Biología y Psicología; las diferencias entre el resto de los grupos no son significativas. Por tanto, parece claro que el grupo de Físicas obtiene una puntuación significativamente mayor que todos los demás, incluyendo al grupo de Químicas (segunda mejor puntuación), y éstos, a su vez, también son mejores que los biológos y psicólogos. De estos resultados se deduce que la especialidad de estudios es el factor que condiciona más la puntuación obtenida, de modo que incluso las diferencias significativas obtenidas para el sexo podrían no ser causadas directamente por esta variable, sino indirectamente por la especialidad (por ejemplo, si existen más mujeres en las especialidades con menores puntuaciones).

Para elucidar esta cuestión, se ha realizado la comparación hombres/mujeres dentro de cada grupo de especialidad (dada la homogeneidad respecto a su formación y su pequeño tamaño, los cuatro últimos grupos de especialidad se han considerado como un sólo grupo). La comparación de las puntuaciones (Tabla II) entre hombres y mujeres dentro de cada especialidad muestra que los hombres obtienen en todos los casos puntuaciones superiores a las mujeres; Ias diferencias no son significativas en la especialidad de Físicas $(p[8,1]=.4638)$, pero son muy próximas al nivel mínimo de significación en Química $(\mathrm{p}[18,1]=.0528)$ y Biología $(\mathrm{p}[39,1\}=$ .0867 ), y resultan muy significativas para el grupo que resume el resto de las especialidades ( $[45,1]=.0034$ ). Por tanto, los hombres tienen puntuaciones mejores que las mujeres en todas las especialidades, y el grado de significación de las diferencias se hace más alto (coeficiente menor) en los grupos a medida que su formación en Física es menor, en el orden Física, Química, Biología y el resto de titulaciones.

A la luz de los análisis anteriores, parece fuera de toda duda la influencia de la especialidad sobre las puntuaciones individuales en el test de concepciones alternativas. Esta importancia de la especialidad para las puntuaciones de cada persona sugiere, por analogía, valorar también la influencia de la especialidad sobre las tasas de aciertos en cada una de las cuestiones planteadas, representadas gráficamente en la figura 1, que permiten apreciar la ejecución diferencial de los diferentes grupos sobre cada cuestión planteada. En esta figura se percibe la superioridad de los resultados del grupo de Físicas en todas las cuestiones, aunque estas diferencias están amortiguadas (diferencias sólo del orden de un $20 \%$ o menos) en las cuestiones 3 (circuito), 5 (inercia) 12 (conservación de energía) y 13 (peso y densidad).

La superioridad del grupo de Químicas respecto a los otros grupos detectada en el ANOVA para las puntuaciones de aciertos aparece un tanto devaluada, ya que la figura muestra que la línea de Químicas coincide prácticamente con Ios otros grupos de menor puntuación, en casi todos los ítems; únicamente aparece superior en los ítems 6 (composición de movimientos), 7 (fuerza peso) $y$ los tres ítems de interacciones $(8,9$ y 10$)$. Teniendo en cuenta que estos tres últimos ítems se refieren al mismo tema, cualitativamente, Ias diferencias del grupo de Químicas respecto a los otros grupos parecen menos importantes.

Las líneas corrrespondientes a biólogos y al resto de grupos son prácticamente coincidentes en todas las cuestiones, de modo que se puede considerar que no existen diferencias entre las concepciones alternativas de los biólogos y el resto de los otros grupos, que no han recibido más formación en física, posiblemente, que la del COU o bachillerato (si cursaron la especialidad de ciencias).

\section{DISCUSIÓN}

Dentro del paradigma de las concepciones alternativas, este estudio examina las diferencias en algunos tópicos de física entre diversas titulaciones específicamente científicas y no científicas (grupo de contraste) con una muestra de aspirantes a profesores (profesores en formación pre-servicio). Los resultados confirman la hipótesis planteada: las titulaciones superiores no concordantes con la física no suministran a los profesores una formación adecuada para superar algunas concepciones alternativas elementales, propias de muchos de sus potenciales alumnos y, por tanto, no se justificaría que estos profesores impartieran física como asignatura afin, sin un riesgo evidente para la calidad de la enseñanza.

Los resultados analizados demuestran con toda nitidez que los aspirantes a profesores tienen una estructura de concepciones alternativas similar en algunos casos a las que tienen sus potenciales alumnos de enseñanza secundaria o bachillerato. El análisis de las concepciones alternativas en fúsica por especialidades de estudios demuestra que sólo los profesores-alumnos de la especialidad de Físicas (especialidad coincidente con el tipo de concepciones alternativas investigadas) no exhiben concepciones alternativas, en tanto que todas las demás especialidades poseen unos niveles de concepciones alternativas en física semejantes a las de los alumnos de 
bachillerato. En el caso de la especialidad de Químicas, aunque un poco mejor que los demás, se ha observado que esta pequeña superioridad se funda en los resultadós de tres cuestiones muy similares, en tanto que en las demás cuestiones ofrece una sensible igualdad con las otras especialidades. La especialidad de Biología tiene una estructura de concepciones alternativas idéntica al grupo de especialidades no científicas (principalmente Psicología y Económicas); es decir, que las concepciones aiternativas en física de un biologo y un psicólogo serían prácticamente idénticas.

Por tanto, la conclusión clara de este análisis de concepciones alternativas en física es que sólo los licenciados en Físicas no cometen los errores conceptuales característicos de las concepciones alternativas, en tanto que las demás especialidades de titulaciones de ciencias no se diferencian significativamente entre sí y muestran un patrón de concepciones alternativas comparable al de sus potenciales alumnos de bachillerato o secundaria. La importancia de este resultado no sólo reside en el corolario obvio que se deduce: el grupo de licenciados en Físicas muestran una mayor aptitud para la docericia de física que todos las demás especialidades, los cuales no se diferencian entre sí. Existe, sin embargo, otra interpretación de este resultado que tiene más alcance y profundidad para la calidad de la enseñanza de las ciencias, basada en la potencia del paradigma de las concepciones alternativas aplicado.

En efecto, este estudio demuestra que las especialidades distintas de Físicas tienen unas concepciones alternativas arraigadas y semejantes a las identificadas en los alumnos. Puesto que son muy resistentes al cambio y forman verdaderas teorías en acción, será difícil eliminarlas, y más si se tiene en cuenta que los profesoresalumnos acaban (o han acabado ya) su periodo de formación científica más importante; en consecuencia, puede esperarse que estas concepciones se transmitan a los alumnos a través de la enseñanza de esos profesores, cuando asuman una docencia que les encomienda unos contenidos integrados $o$ asignaturas afines.

Esta situación podría tener dos soluciones: cambiar las decisiones administrativo-laborales que asignan asigna-

\section{REFERENCIAS BIBLIOGRÁFICAS}

ABIMBOLA, I.O., 1988. The Problem of Terminology in the Study of Student Conceptions in Science, Science Education, 72, pp. $175-184$.

ACEVEDO, I.A., BOLÍVAR, J.P., LÓPEZ-MOLINA, E.J. y TRUJILLO, M., 1989. Sobre las concepciones en dinámica elemental de los adolescentes formáles y concretos y el cambio metodołógico, Enseñanza de las Ciencias, 7, pp. 27-34. turas afines a los profesores o modificar profundamente la formación pre-servicio y dentro del servicio del profesorado, salidas que aunque parecen independientes, en la toma de decisiones van unidas, ya que una suele condicionar la otra.

Puesto que lo más importante es conseguir una formación adecuada de los profesores de ciencias, parece que Ia salida prioritaria debería enfocarse por diseñar una formación del profesor de ciencias adecuada a los objetivos y contenidos de la enseñanza. Actualmente existe una tendencia a enfatizar la importancia, en el perfil del profesor, de la capacitación para las tareas de tipo psicopedagógico, comunes a todos los profesores y en todos los niveles (tutorías, organización, diseño curricular, atención a la diversidad, desarrollo de temas transversales, etc.), en detrimento indirecto de la formación especializada en las áreas de conocimiento. Una muestra de ello es la reorganización de la formación de los profesores de primaria, sustituyendo las anteriores especialidades (Sociales, Ciencias, Lengua...) por una única especialidad (maestro de primaria), apareciendo otras nuevas, acordes con la filosofía anterior. En la eđucación secundaria y en el bachillerato, ta formación pre-servicio está en manos de las universidades y sus nuevos planes de estudios, ya que los profesores deben ser titulados superiores. Pero también la formación en servicio ofrecida por el $\mathrm{MEC}$ a los profesores, a lo largo de su vida de ejercicio profesional, tiene un importante desafío.

Sea cual sea el diseño adoptado, y ya que los proyectos de ciencia integrada en la enseñanza obligatoria y la docencia en asignaturas afines en bachillerato deben aceptarse como situaciones reales en la enseñanza, se requiere mejorar y profundizar la formación de los profesores, tanto en la formación inicial como en la formación en servicio, con una dirección muy clara y prioritaria para los programas de formación de profesorado de ciencias: promover el cambio conceptual y remover las concepciones alternativas del esquema conceptual de los profesores, como condición para la calidad en la enseñanza de las ciencias.
BULLEIOS, J. y SAMPEDRO, C., 1990 . Diferenciacion de los conceptos de masa, volumen y densidad en alumnos de BUP, mediante estrategias de cambio conceptual y metodológico, Enseñanza de las Ciencias, 8, pp. 31-36.

CARAMAZZA, A., MCCLOSKEY, M. y GREEN, B., 1981 Naive beliefs in «sophisticated» subjects: misconceptions about trajectories of objects, Cognition, 9, pp. 117-128. 
CARRASCOSA, J. y GIL, D., 1982. Los errores conceptuales en la enseñanza de la Física. Un estudio de su persistencia. Primeras Jornadas de Investigación en Didáctica en Física y Química, ICE Valencia, pp. 277-281.

CARRASCOSA, J. y GIL, D., 1985. La «metodología de la superficialitat» i l'aprenentatge de les ciències, Enseñanza de las ciencias, 3, pp. 113-120.

CARRASCOSA, J. y GIL, D., 1987. Diferencias en la evolución de preconceptos de Mecánica y Química. Comunicación presentada ai II Congreso Internacional sobre investigación en la Didáctica de las Ciencias y Matemáticas, Valenciá.

CHAMPAGNE, A., GUNSTONE, R. y KLOPFER, L., 1983. Effecting changes in cognitive structure amongst physics students. Paper presented at the Annual Meeting of the American Association (Symposium Stability and Change in Conceptual Understanding), Montreal.

COHEN, R., EYLON, B. y GANIEL, U., 1983. Potential difference and current in simple electric circuits: A study of students' concepts, American Journal of Physics, 51, pp. 407-412.

CUBERO, R., 1988. Los marcos conceptuales de los alumnos como esquemas de conocimiento. Una interpretación cognitiva, Investigación en la escuela, 4, pp. 3-11.

DE VEGA, M., 1983. Fílogénesis, adaptación y sesgos biológicos del conocimiento: una altemativa a las analogías formales, Boletín de Psicología, I, pp. 11-149.

DRIVER, R. y EASLEY, J., 1978. Pupils and paradigms: A review of literature related to concept development in adolescent science studies, Studies in Science Education, 5, pp. 6I-84.

DRIVER, R. y ERICKSON, G, 1983. Theortes in action: Some theoretical and empirical issues in the study of students" conceptual frameworks in science, Studies in Science Education, 10. pp. $37-60$.

DRIVER, R., GUESNE, E. y TIBERGHIEN, A., 1989. ldeas científicas en la infancia y en la adolescencia. (MEC-Morata: Madrid).

FERNÁNDEZ, M.C. y VÁZQUEZ, A., 1991. Evaluación inicia] y construcción del aprendizaje: Análisis de una experiencia con profesores en formación [Resumen]. Programa del III Congreso sobre el Pensamiento del Profesor y el Desarrollo Profesional, p. 84. Sevilla: Universidad de Sevilla (26-28 junto).

GIL, D., 1987. Los errores conceptuales como origen de un nuevo modelo didáctico: de la búsqueda a la investigación, Investigación en la escuela, 1 , pp. 35-41.

HASHWED, M.Z., 1986. Towards an explanation of conceptual change, European Journal of Sciencie Education, 3, pp. 383-396.
HELM, H., 1980. Misconceptions in Physics among South African Students, Physics Education, 15, pp. 92-105.

HELM, H. y NOVAK, J.D., 1983. Proceedings of the International Seminar on Misconceptions in Science and Mathematics. (Cornell University: Ithaca. Nueva York).

HEWSON, P.W., 1981. A conceptual Change Aproach to Learning Sciencie, European Journal of Sciencie Education, 3, pp. 383-396.

HIERREZUELO, J, y MOLINA, E, , 1990. Una propuesta para la introducción del concepto de energía en el bachillerato, Enseñanza de las Ciencias, 8, pp. 23-30.

HIERREZUELO, J. y MONTERO, A., 1988. La ciencia de los alumnos. (MEC-Laia: Barcelona).

LEHRMAN, R.L., 1982. Confused physics: A tutorial critique, The Physics Teacher, 20, pp. 519-523.

MCDERMOTT, L.C., 1984. An overview of research on conceptual understanding in mechanics, Physics Today, 37, pp. 7-24.

NUSSBAUM, J., 1981. Towards the Diagnosis by Science Teachers of Pupil's Misconceptions: An Exercise with Student Teachers, European Journal of Sciencie Educution, 3, pp. $159-169$.

OSBORNE, R.J., BELL, B.F. y GILBERT, J.K., 1983. Science teaching and children's views of the world, European Journal of Sciencie Education, 5, pp. 1-14.

OSBORNE, R. y FREYBERG, P., 1985. Learning in Science. (Heinemann: Porstmouth NH).

POZO, J.I. y CARRETERO, M., 1987. Del pensamiento formal a las concepciones espontáneas. ¿Quécambia en la enseñanza de la ciencia?, Infancia y aprendizaje, 38, pp. 35-52.

SEBASTIÀ, J.M., 1984. Fuerza y movimiento: la interpretación de los estudiantes, Enseñanza de las Ciencias, 2, pp. $161-169$.

VÁZQUEZ, A., 1990. Concepciones alternativas en física y química de bachillerato: una metodologia diagnóstica, Enseñanza de las Ciencias, 8, pp. 251-258.

VIENNOT, L., 1979. Le raisonnement spontané en dinamique élementaire. (Hermann: París).

WHITAKER, R.J., 1983. An examination of student inconsistencies in their understanding of trajectory motion, en H. Helm y J. Novak (eds.), Misconceptions in science and mathematics. (Cornell University: Ithaca, Nueva York). 


\section{ANEXO}

1. ¿Cuál de las tres posiciones: A, B O C, adoptaría el sistema inicial después de dejarlo libre?

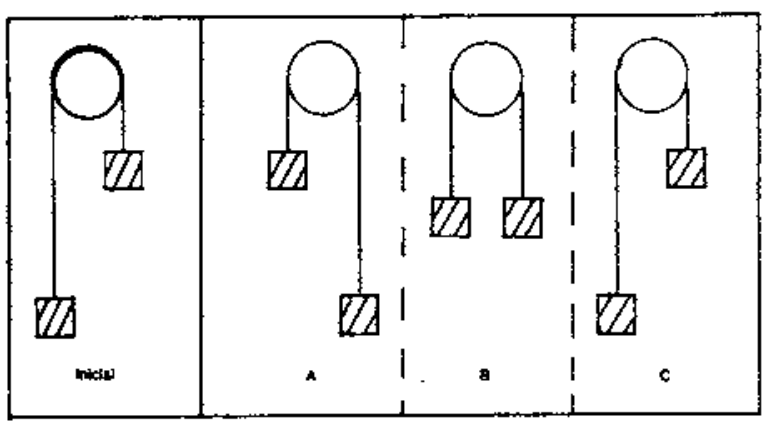

2. Un cuerpo ha sido lanzado y está subiendo libremente por el plano inclinado. ¿Cuál de los tres dibujos representa correctamente la fuerza resultante que está actuando sobre el cuerpo?

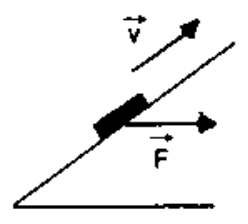

A

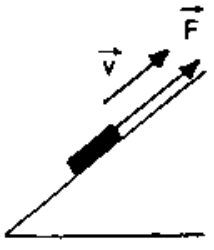

B

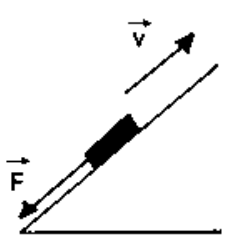

c.

3. La pila de la figura no tiene resistencia interna. Las bombillas $\mathrm{M}$ y $\mathrm{N}$ están encendidas. Quitamos $\mathrm{N}$ del casquillo. Consecuentemente:

a) La bombilla M lucirá más fuertemente.

b) La d.d.p. entre D y E será cero.

c) La d.d.p. entre D y E no cambiará.

d) La d.d.p. entre D y E aumentará.

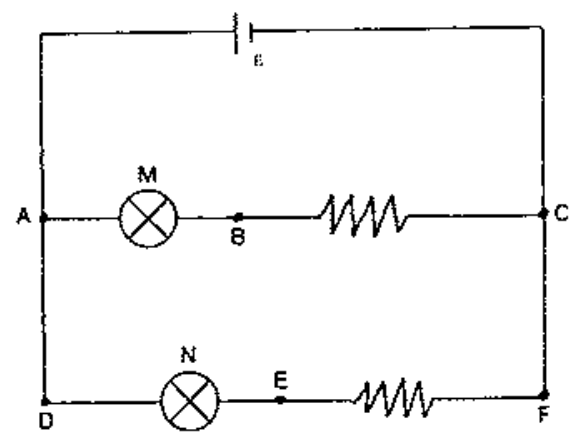

4. En el punto A, cuando la pelota está subiendo, ¿qué dibujo crees que representa mejor la fuerza sobre la pelota?
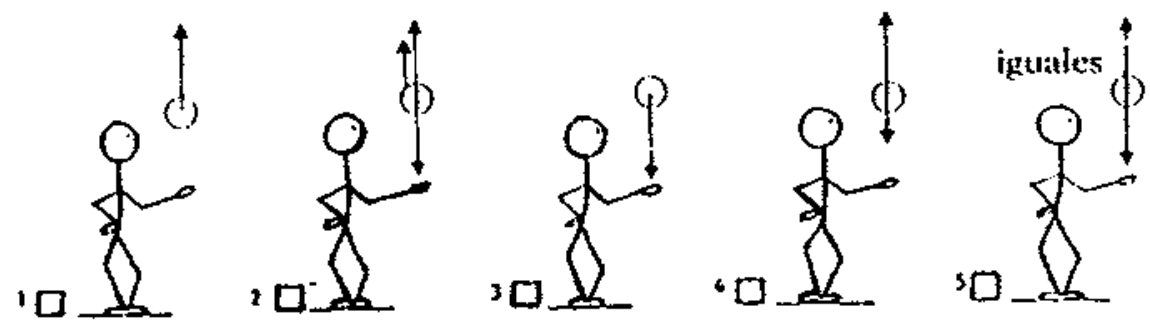
5. Un tren circula por una vía recta a la velocidad de $70 \mathrm{~km} / \mathrm{h}$. En un vagón hay un pequeño agujero en el suelo y en el techo, justo encima del agujero, hay un tornillo. El tornillo se suelta y cae. ¿Cuâl de las siguientes opciones te parece correcta?

a) El tornillo cae al suelo delante del agujero.

b) El tornillo cae y pasa por el agujero.

c) El tornilio cae detrás del agujero.

6. En una lianura disparamos una bala horizontalmente desde una altura de $10 \mathrm{~m}$. Al mismo tiempo dejamos caer desde la misma aitura una bola de hierro, teniendo cuidado de que el momento de salida de la bala y el de caída de la bola coincidan. La bola llegará ai suelo:

a) Antes que la bala.

b) Al mismo tiempo que la bala.

c) Después de la bala.

7. Un jugador malabarista juega con 6 bolas idénticas. En un determinado momento, las seis bolas están a la misma altura, con trayectorias como las de la figura. También hemos dibujado los vectores velocidad en ese momento. Las fuerzas que actúan sobre las bolas, ¿son iguales o diferentes?
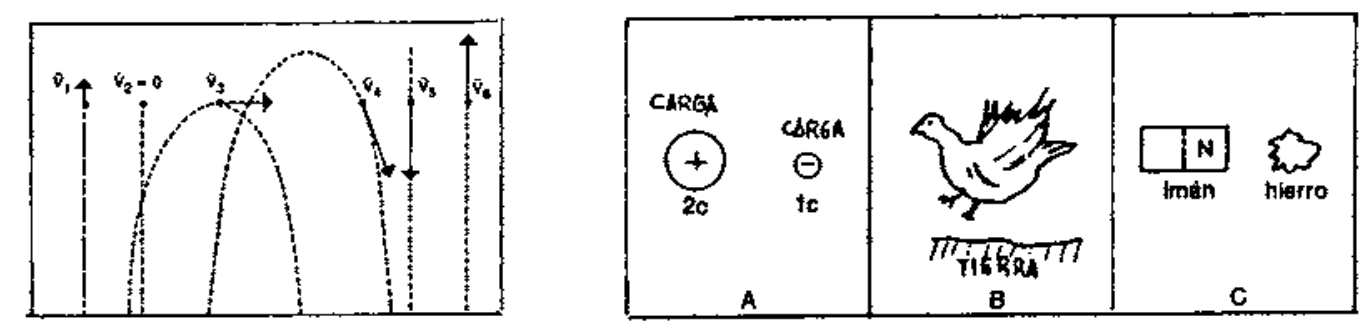

8. En cada una de las viñetas, $A, B$ y $C$, ¿quién atrae a quién?

9. En la figura se representa la trayectoria circular de la Luna altededor de la Tierra. ¿Qué fuerzas actúan sobre la Luna?

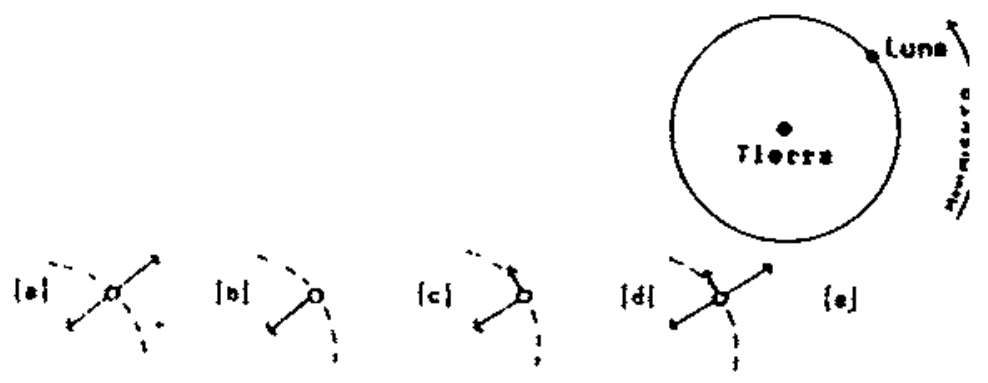


10. Un coche liega al principio de una cuesta con una determinada velocidad. En ese momento se le para el motor, pero aún puede subir hasta lo alto de la cuesta, lugar donde se detiene.

Señala qué frase te parece correcta, explicando tu respuesta:

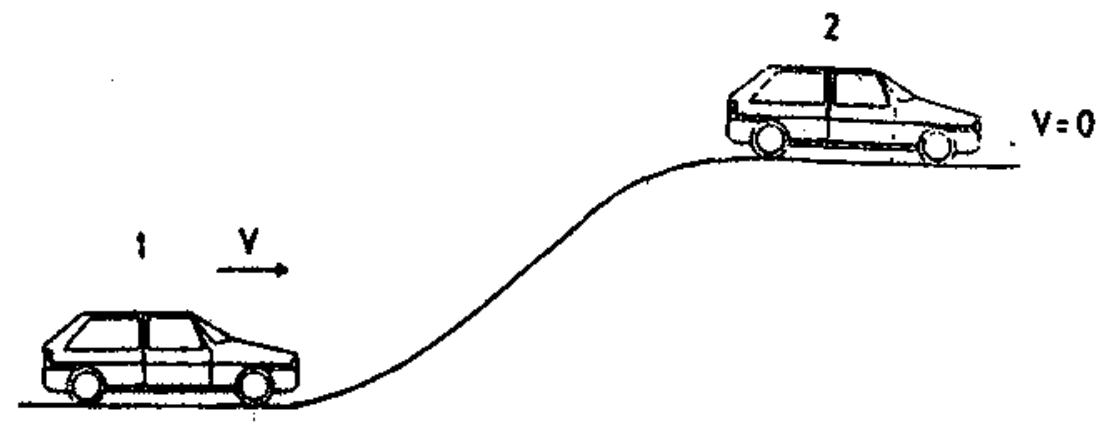

Respecto a la energia:

a) Tiene más energía en 1 .

b) Tiene más energía en 2 .

c) Tiene la misma energía en 1 que en 2 .

d) Ninguna es correcta.

11. Dos objetos sólidos, A y B, se sumergen cada uno en sendos vasos con agua. El nivel del agua en cada vaso, antes y después de la inmersión, se muestra en la figura.

Antes de la inmersión

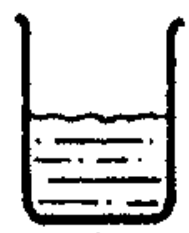

A.

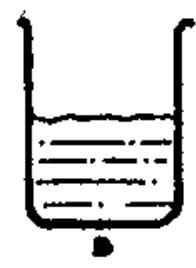

Después de la inmersión
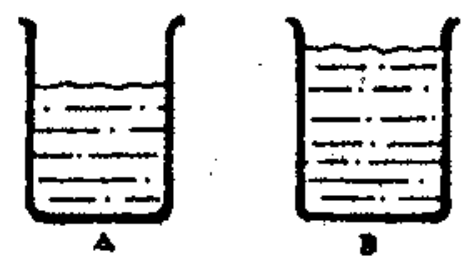

Del peso de estos dos objetos podemos decir:

a) Peso de $\mathrm{A}=$ Peso de $\mathrm{B}$

b) Peso de $A<$ Peso de $B$

c) Peso de $A>$ Peso de $B$

d) Asi no podemos sáber nada del peso. 